\title{
Noise and aggregation of information in large markets*
}

\author{
Diego García Branko Urošević $^{\ddagger}$
}

October 8, 2004

\begin{abstract}
We study a novel class of noisy rational expectations equilibria in markets with large number of agents. We show that, as long as noise increases with the number of agents in the economy, the limiting competitive equilibrium is well-defined and leads to non-trivial information acquisition, perfect information aggregation, and partially revealing prices, even if per capita noise tends to zero. We find that in such equilibrium risk sharing and price revelation play different roles than in the standard limiting economy in which per capita noise is not negligible. We apply our model to study information sales by a monopolist, information acquisition in multi-asset markets, and derivatives trading. The limiting equilibria are shown to be perfectly competitive, even when a strategic solution concept is used.
\end{abstract}

JEL classification: D82, G14.

Keywords: partially revealing equilibria, competitive equilibrium, rational expectations, information acquisition, markets for information, derivatives trading, multi-asset markets, share auctions.

\footnotetext{
*A significantly shorter version of this paper circulated under the title "Noise and aggregation of information in competitive rational expectations models." We would like to thank Andrew Bernard, Peter DeMarzo, Hayne Leland, Rodolfo Prieto, Francesco Sangiorgi, Brett Trueman, Joel Vanden and Dimitri Vayanos for comments on an early draft, as well as seminar participants at Dartmouth College, HEC, the XXVIII Simposio de Análisis Económico, and at the 2004 WFA meetings. All remaining errors are our own. The latest version of this paper can be downloaded from http://diego-garcia.dartmouth.edu.

${ }^{\dagger}$ Correspondence information: Diego García, Tuck School of Business Administration, Hanover NH 037559000, US, tel: 1-603-646-3615, fax: 1-603-646-1308, email: diego.garcia@Dartmouth.edu.

${ }^{\ddagger}$ Branko Urošević, Department of Economics and Business, Universitat Pompeu Fabra, 08005, Barcelona, Spain, tel: 34-93-542-2590, email: branko.urosevic@upf.edu. Also affiliated with CREA (Barcelona) and SECCF (Belgrade).
} 


\section{Introduction}

Ever since the seminal work of Grossman (1976) and Grossman and Stiglitz (1980), the informational content of prices in competitive market settings has been a subject of significant interest in economics as well as in different areas of applied research. ${ }^{1}$ As emphasized in Black (1986), noise plays a crucial role in preventing prices of traded assets from fully revealing agents' private information. ${ }^{2}$ The purpose of this paper is to study the effects that the level of noise plays in the acquisition, revelation and aggregation of private information in competitive markets. By decoupling the concepts of a large number of agents and a large level of noise, and allowing for endogenous information acquisition of costly private signals, we uncover a new class of tractable competitive rational expectation equilibria (REE) that generalizes previously known results. We find that in order for prices not to fully reveal private information the level of noise and the size of the informed trader population must be of the same order of magnitude, as measured by the total number of agents in the economy. We demonstrate that even if per capita noise is negligible a well-defined competitive equilibrium exists. In such equilibrium, the endogenously determined number of informed traders is negligible in comparison to the total trader population, and, as a result, prices only partially reveal private information. We refer to this novel class of equilibria as the "diversifiable endowment risk model."

We compare and contrast this new class of equilibria with the standard model (see Hellwig (1980) and Verrecchia (1982)). In that model, which we refer to as the "systematic endowment risk model", per-capita noise in the large economy is positive and, as a result, the equilibrium fraction of informed traders is positive as well. ${ }^{3}$ Models with systematic and diversifiable risk have some important features in common. In particular, prices in both are strictly partially revealing. ${ }^{4}$ In addition, prices perfectly aggregate information, i.e. individual signals do not show up in the price function (Hellwig (1980)). ${ }^{5}$ Yet another similarity between the two models is that in terms of information revelation in both models the informed agents are marginal even

\footnotetext{
${ }^{1}$ In Finance, rational expectations models have been used to study markets for information (Admati and Pfleiderer (1986), Admati and Pfleiderer (1990)), derivatives (Brennan and Cao (1996), Cao (1999)), insider trading (Ausubel (1990a), Bushman and Indjejikian (1995)), security design problems (Duffie and Rahi (1995), Demange and Laroque (1995), Rahi (1996)), and the dynamics of asset prices and volume (Campbell, Grossman, and Wang (1993), Wang (1993)), among other topics. In Accounting, many issues around disclosure and compensation have been studied within the standard rational expectations paradigm (see, for example, Diamond and Verrecchia (1982), Diamond (1985), Banker and Datar (1989), Bushman and Indjejikian (1993), Kim and Verrecchia (1991) and the references in Verrecchia (2001)).

${ }^{2}$ Interpretation of a source of noise may vary from model to model: noise can be the result of noise traders' demand or agents' aggregate endowment shocks, for example. While the exact interpretation of noise is immaterial in what follows, we frequently use the latter interpretation.

${ }^{3}$ Other tractable REE models of financial markets can be found, for example, in Ausubel (1990b) and DeMarzo and Skiadas (1998).

${ }^{4}$ By this we mean that the conditional variance of the final payoff of the risky asset, given the asset price, is strictly greater than zero and strictly less than the unconditional variance of the payoff.

${ }^{5}$ In other words, as the number of agents in the economy grows without bound, the price of a risky asset depends only on its final payoff and on a variable that parameterizes the risky aggregate supply.
} 
though the size of the informed population is negligible in the diversifiable risk model and not negligible in the systematic risk model.

Despite these similarities, there are also significant differences between the two classes of models. In particular, since in the diversifiable risk model the equilibrium fraction of informed agents vanishes in the limit, risk sharing and price revelation play a different role than in the standard model. As a result, in the diversifiable risk model only the most risk-tolerant agents become informed, while less risk-tolerant agents never do. In such an economy, in terms of risksharing capacity the marginal trader is an uninformed agent. On the other hand, populations of both informed and uninformed traders are not negligible in the systematic risk model and both contribute to the risk sharing capacity in the economy (see Verrecchia (1982)). This difference drives a wedge between the two models in terms of the shape of the risk premia, as well as several important comparative statics.

We, then, compare and contrast the two models in three applications from the literature, namely in studies of markets for information (Admati and Pfleiderer (1986)), information acquisition in multi-asset securities markets (Admati (1985)), and derivatives trading (Brennan and Cao (1996)). We show that a monopolist seller of information will have very different types of optimal sales in the diversifiable and systematic-risk models. In the systematic risk case information monopolist would optimally sell information to the whole population adding personalized noise to her private signal in order to prevent full information revelation. In the diversifiable risk case, the optimal strategy involves selling a signal of the smallest possible precision to a proper fraction of the population. In our analysis of information acquisition in multi-asset markets, we study a model where one asset has systematic risk and the other has diversifiable risk. We show that the incentives to gather information on the asset with systematic risk can be significantly altered once the diversifiable asset is introduced. Moreover, when there are complementarities in the information acquisition technology, the equilibrium significantly differs from that in which both assets have systematic risk. As our third application, we study an equilibrium with derivatives. While there are differences in terms of functional forms in the diversifiable and systematic risk model, some of the qualitative aspects of the solution are similar in both types of large markets.

The early papers by Hellwig (1980), Diamond and Verrecchia (1981) and Verrecchia (1982) are closest to our work, both in terms of the motivation and the model setup. Our diversifiablerisk equilibrium follows the setup in Diamond and Verrecchia (1981), endogenizing, in addition, the information acquisition decisions as in Verrecchia (1982). The literature on aggregation of information in auction settings is also related. ${ }^{6}$ There are relatively few papers that consider

\footnotetext{
${ }^{6}$ See Wilson (1977), Milgrom (1979), and Milgrom (1981) for some of the early references. Recent work in this area includes Pesendorfer and Swinkels (1997), Pesendorfer and Swinkels (2000), Kremer (2002), Hong and Shum (2004), Jackson and Kremer (2004).
} 
the endogenous acquisition of information in such settings (see Matthews (1984) and Jackson (2003)). ${ }^{7}$ In order to make an explicit connection with the auctions literature we extend the model by allowing agents to act strategically, as in Kyle (1989). We show that the perfectly competitive equilibria in diversifiable and systematic risk economies can be seen as limiting economies of such strategic model. ${ }^{8}$ Since the market described in Kyle (1989) can be viewed as a share auction market, our paper also contributes to the literature on large auction markets. In particular, the paper provides necessary and sufficient conditions on the level of noise in the economy needed to support endogenous acquisition of information in a particular type of auction environment.

The structure of the paper is as follows. In section 2 we present our main model based on the assumptions of agents' homogeneity and the existence of an exogenous random aggregate supply shock (or, equivalently, noise traders). Section 3 is the central section of the paper and contains its main results. Section 4 studies the applications of the diversifiable risk model to problems of sales of information, information acquisition in multi-asset markets, and derivatives trading, and compares the results with those obtained in the standard systematic risk framework. Section 5 considers various extensions of the basic model and shows that the main results of the paper are robust. Proofs are collected in the Appendix.

\section{The finite-agent model}

Consider a symmetric one period economy with $N$ traders. We assume that all agents have CARA preferences with a risk aversion parameter $\tau .{ }^{9}$ Thus, given a final payoff $W_{i}$, each agent $i$ derives the expected utility $\mathbb{E}\left[u\left(W_{i}\right)\right]=\mathbb{E}\left[-\exp \left(-\tau W_{i}\right)\right]$. There are two assets in the economy: a risk-less asset in perfectly elastic supply, and a risky asset with a random final payoff $X \in \mathbb{R}$ and variance normalized to 1 . All random variables in the economy are defined on some probability space $(\Omega, \mathcal{F}, \mathbb{P})$, and unless stated otherwise, are normally distributed, uncorrelated, and have zero mean. The risky asset is in a random aggregate supply $Z_{N}$. This variable, which we will refer to loosely as noise, is the main driver in preventing private information to be revealed perfectly to other market participants.

One of the key assumptions of the model is that agents endogenously decide, in a process described below, whether or not to become informed, i.e. to purchase costly private signal of the form $Y_{i}=X+\epsilon_{i}$, where $\operatorname{var}\left(\epsilon_{i}\right)=\sigma_{\epsilon}^{2}$, for all $i$. We let $m_{N} \leq N$ denote the number of agents that decide to become informed. Without loss of generality we can label the informed

\footnotetext{
${ }^{7}$ Vives (1988) studies the aggregation of information in a Cournot-type product market and also studies information acquisition decisions.

${ }^{8}$ While in spirit the convergence results are similar to the results of Section 9 of Kyle (1989), in our case the size of the informed population is, in contrast to Kyle (1989), determined endogenously .

${ }^{9} \mathrm{We}$ allow for heterogeneous risk aversion in section 5 .
} 
agents with the subscripts $1, \ldots, m_{N}$, and the uninformed with the subscripts $m_{N}+1, \ldots, N$. We use $\theta_{i}$, for $i=1, \ldots, N$, to denote the trading strategy of agent $i$, i.e. the number of shares of the risky asset that agent $i$ acquires. With this notation, the final wealth for an agent of type $i$ is given by $W_{i}=\theta_{i}\left(X-P_{N}\right)$, where $P_{N}$ denotes the price of the risky asset in the economy with $N$ agents. ${ }^{10}$ We use $\mathcal{F}_{i}$ to denote the information set at the time of trading of an agent of $i .{ }^{11}$

A rational expectations equilibrium, fixing the number of informed agents $m_{N}$, is characterized by a set of trading strategies $\left\{\theta_{i}\right\}_{i=1}^{N}$ and a price function $P_{N}: \Omega \rightarrow \mathbb{R}$ such that:

(1) Each agent $i$ chooses her trading strategy $\theta_{i}$ so as to maximize her expected utility conditional on her information set $\mathcal{F}_{i}$, i.e.

$$
\theta_{i} \in \arg \max _{\theta} \mathbb{E}\left[u\left(W_{i}\right) \mid \mathcal{F}_{i}\right] \quad i=1, \ldots, N
$$

(2) Markets clear, i.e.

$$
\sum_{i=1}^{N} \theta_{i}=Z_{N}
$$

We remark that agents act as price takers in (1), an assumption which we will revisit in section 5.1. As is customary in the literature, we conjecture that the equilibrium price is linear in the signals and the aggregate random supply. Given the symmetry of the economy this conjecture implies that prices are described by two parameters $b_{N}$ and $d_{N}$, namely

$$
P_{N}=b_{N} \sum_{i=1}^{m_{N}} Y_{i}-d_{N} Z_{N}
$$

At the information gathering stage each agent can acquire a signal $Y_{i}$ at the cost (measured in units of account) of $c>0$. Therefore, upon acquisition, an agent's expected utility reads $\mathbb{E}\left[u\left(W_{i}-c\right)\right]$. It should be noted here that this expectation is unconditional, i.e. that it is taken before the signals are realized. In addition, it presumes that the agents anticipate the rational expectations equilibrium price given in (3). Due to the symmetry of the model, we determine the Nash equilibrium at the information acquisition stage, characterized by $m_{N}$ agents becoming informed, by simply equating the ex-ante expected utilities of informed and uninformed agents.

\footnotetext{
${ }^{10}$ We normalize here, as is customary in the literature, the agents' initial wealth and the risk-free rate to zero. These assumptions are innocuous since the model contains only one period of trading. In addition, there are no borrowing or lending constraints imposed on the agents.

${ }^{11}$ Note that $\mathcal{F}_{i}=\sigma\left(Y_{i}, P_{N}\right)$ for $i=1, \ldots, m_{N}$, and $\mathcal{F}_{i}=\sigma\left(P_{N}\right)$ for $i=m_{N}+1, \ldots, N$, where $\sigma(X)$ denotes the $\sigma$-algebra generated by a random variable $X$.
} 
Thus far we have described a sequence of economies, which we will denote by $\left\{\mathcal{E}_{N}\right\}_{N=1}^{\infty}$, characterized by the primitives $\left(\tau, \sigma_{\epsilon}^{2}, c, Z_{N}\right)$, with associated equilibrium prices $P_{N}$ and informed agents $m_{N}$. Since the focus of the paper is on aggregate noise, we formalize in the next definition the assumptions we make on $Z_{N}$.

Definition 1. We say that a sequence of economies $\mathcal{E}_{N}$ has large noise if there exist $\beta>0$ and $\sigma_{z}^{2}>0$ such that

$$
\lim _{N \uparrow \infty} \operatorname{var}\left(N^{-\beta} Z_{N}\right)=\sigma_{z}^{2} .
$$

If $\beta=1$, we say that the sequence of economies has systematic risk. If $\beta \in(0,1)$, we will use the term diversifiable risk.

Among all sequences of economies with unbounded noise, this definition makes a distinction among those which have finite per-capita risk, the case $\beta=1$, and those with zero per-capita risk, the case $\beta \in(0,1)$. In order to motivate these choices, let aggregate noise be the sum of $N$ i.i.d. random variables. In particular, let $Z_{N}=\sum_{i=1}^{N} Z_{i}$, for a set of random variables $\left\{Z_{i}\right\}_{i=1}^{N}$. If the individual shocks $\left\{Z_{i}\right\}_{i=1}^{N}$ are i.i.d. with constant variance, it is simple to verify that (4) holds for $\beta=1 / 2$. Aggregate noise in this case grows with the number of agents, but it is negligible on a per capita basis. On the other hand, if the random variables $\left\{Z_{i}\right\}_{i=1}^{N}$ are sufficiently correlated with each other, one can verify that (4) holds with $\beta=1 .{ }^{12}$ The names diversifiable and systematic are borrowed from portfolio theory in a natural way.

This completes the description of the model. In order to emphasize the dependence of equilibrium variables on the type of noise they possess (systematic or diversifiable), we will use the notation $\mathcal{E}_{N}(\beta), P_{N}(\beta)$ and $m_{N}(\beta)$ to denote the sequence of economies, prices and number of informed agents respectively. In a slight abuse of notation, we let $P_{\beta} \equiv \lim _{N \uparrow \infty} P_{N}(\beta)$ denote the limiting prices. ${ }^{13}$ Lastly, we say that a sequence of economies $\mathcal{E}_{N}(\beta)$ has a partially revealing $R E E$ if $\operatorname{var}\left(X \mid P_{\beta}\right) \in(0,1)$, i.e. if prices neither fully reveal information nor are they completely uninformative.

\footnotetext{
${ }^{12}$ Verrecchia (1982) motivates the systematic risk model by assuming the individual shocks $\left\{Z_{i}\right\}_{i=1}^{N}$ are i.i.d. with variance that grows linearly in $N$. This reduces to a limiting economy satisfying (4) for $\beta=1$. Also, if the shocks $\left\{Z_{i}\right\}_{i=1}^{N}$ are identically distributed, but with non-trivial correlation with each other, (4) also holds for $\beta=1$.

${ }^{13}$ One technical caveat is in order. In the next section we will discuss convergence properties of the sequence of prices $P_{N}$. The reader should bear in mind that all convergence statements there are both a.s. as well as in $\mathcal{L}^{2}$, although, for brevity, we omit these qualifiers in what follows.
} 


\section{The limiting equilibria in large competitive economies}

This section contains some of the key results of the paper. We first establish the existence of a limiting rational expectations equilibria with endogenous information acquisition in both the diversifiable and the systematic risk models. We then discuss qualitative differences between the two models.

\subsection{Partially-revealing rational expectations equilibria}

We begin our analysis by establishing an important preliminary result: prices in a limiting economy are partially revealing if and only if the number of informed agents grows at the same rate as the level of noise in the economy.

Lemma 1. Consider a sequence of economies $\mathcal{E}_{N}(\beta)$, where $\beta \in(0,1]$. Suppose that the endogenously determined number of informed agents $m_{N}$ satisfies

$$
\lim _{N \rightarrow \infty} N^{-\alpha} m_{N}(\beta)=\lambda_{\alpha}
$$

for some positive real numbers $\alpha$ and $\lambda_{\alpha}$. A necessary and sufficient condition for prices in the limiting economy to be partially revealing is that $\alpha=\beta$. Furthermore, when $\alpha=\beta$ there exist some positive constants $a_{\beta}, d_{\beta}$ such that:

$$
P_{\beta}=a_{\beta} X-d_{\beta} Z_{\beta}
$$

where $Z_{\beta} \equiv \lim _{N \rightarrow \infty} N^{-\beta} Z_{N}$.

The lemma states that in order for prices in the limiting economy to be partially revealing, it is necessary for $m_{N}$ to grow at the rate $\beta$, i.e. at the same rate as the standard deviation of the aggregate noise $Z_{N}$. In order to gain some intuition for this result, it is useful to re-write the price function in the finite-agent economy (3) as follows:

$$
P_{N}=d_{N} N^{\beta}\left(N^{-\beta} m_{N}\left(\frac{b_{N}}{d_{N}}\right) \bar{Y}_{N}-N^{-\beta} Z_{N}\right)
$$

where $\bar{Y}_{N}=m_{N}^{-1} \sum_{i=1}^{m_{N}} Y_{i}$. Price in the finite-agent economy is a weighted average of the sum of the signals received by the agents, $\bar{Y}_{N}$, and a random variable, $N^{-\beta} Z_{N}$, that parameterizes the aggregate supply shocks. Further note that this "average noise" term $N^{-\beta} Z_{N}$ has a nondegenerate limit in economies with large noise, namely the random variable $Z_{\beta}$ defined in the lemma, which from Definition 1 satisfies $\operatorname{var}\left(Z_{\beta}\right)=\sigma_{z}^{2}$. The lemma establishes that the relative price coefficients $b_{N} / d_{N}$ converge to a positive limit for large $N$; from (7) it follows that the 
existence of a finite limit for $N^{-\beta} m_{N}$ is a necessary and sufficient condition for prices to be partially revealing.

The following theorem represents the central result of the paper.

Theorem 1. Consider a sequence of economies $\mathcal{E}_{N}(\beta)$, where $\beta \in(0,1]$. Let $C \equiv e^{2 \tau c}-1$, and assume that $C^{-1}>\sigma_{\epsilon}^{2}$. In a limiting information acquisition equilibrium asset prices are partially revealing and perfectly aggregate private information. In particular, the price function is given by (6) and the equilibrium number of informed traders satisfies

$$
\lim _{N \rightarrow \infty} N^{-\beta} m_{N}(\beta)=\lambda_{\beta}
$$

for some $a_{\beta}, d_{\beta}, \lambda_{\beta}>0$.

(i) In the systematic risk model, where $\beta=1$, the price coefficients satisfy:

$$
a_{1}=\lambda_{1} r_{1} d_{1} ; \quad d_{1}=\frac{1+\frac{\lambda_{1} r_{1}}{\tau \sigma_{z}^{2}}}{\lambda_{1} r_{1}+\frac{1}{\tau}\left(1+\frac{\left(\lambda_{1} r_{1}\right)^{2}}{\sigma_{z}^{2}}\right)} ; \quad r_{1}=\frac{1}{\tau \sigma_{\epsilon}^{2}}
$$

Moreover, if $C^{-1}<\sigma_{\epsilon}^{2}\left(1+r_{1}^{2} / \sigma_{z}^{2}\right)$ then

$$
\lambda_{1}=\tau \sigma_{z} \sigma_{\epsilon} \sqrt{C^{-1}-\sigma_{\epsilon}^{2}}
$$

otherwise $\lambda_{1}=1$.

(ii) In the diversifiable risk model, where $0<\beta<1$, the price coefficients are given by

$$
a_{\beta}=\lambda_{\beta} r_{\beta} d_{\beta} \quad d_{\beta}=\frac{\lambda_{\beta} r_{\beta} / \sigma_{z}^{2}}{1+\frac{\left(\lambda_{\beta} r_{\beta}\right)^{2}}{\sigma_{z}^{2}}} ; \quad r_{\beta}=\frac{1}{\tau \sigma_{\epsilon}^{2}}
$$

Moreover, when $0<\beta<1, \lambda_{\beta}=\lambda_{1}$, as given in (10).

Theorem 1 formalizes the intuitive idea that in order to have a well defined limiting economy with endogenous information acquisition decisions, the conditions in Lemma 1 must be satisfied. Moreover, the Theorem characterizes the limiting prices $P_{\beta}$ in closed-form for both the systematic and diversifiable risk models. We remark that the condition $C^{-1}>\sigma_{\epsilon}^{2}$ simply rules out limiting equilibria in which prices are uninformative and all agents optimally stay uninformed.

The parameter $\lambda_{\beta}$ in (8) measures the amount of informed trading per unit of noise. In the systematic risk model, $\lambda_{1}$ corresponds to the fraction of agents that becomes informed. In the diversifiable risk model, the equilibrium fraction of informed agents goes to zero at the 
rate $N^{-(1-\beta)}$. However, the amount of informed agents per unit of noise in this case is not zero and is given by $\lambda_{\beta}$. In equilibrium, the "right" mass of agents becomes informed and the asset price acts as an aggregator of information possessed by the traders. It is interesting to note that in the systematic risk case there are economic primitives under which all agents in the economy become informed $\left(\lambda_{1}=1\right)$. This can never occur in economies with diversifiable risk, since the population of informed traders in that case is negligible in size with respect to the total number of agents in the economy $N$.

We conclude this section by mentioning that when either $\beta=0$ or $\beta>1$ in Definition 1 limiting information acquisition equilibrium may exist, but would have undesirable properties. In particular, when $\beta=0$ only a finite number of agents becomes informed in equilibrium, and, therefore, individual signals show up in the expression for the equilibrium price making the price aggregation of information imperfect, as well as hindering the tractability of the model. On the other hand, if $\beta>1$ noise grows "too fast": one can check that in this case limiting prices would reveal no information about the fundamental value of the asset, even if all agents would choose to become informed.

\subsection{Comparing the systematic and diversifiable risk models}

In this section we compare systematic and diversifiable risk limiting information acquisition equilibria along three economic characteristics: price informativeness, the effect of the introduction of asymmetric information, and price volatility. As customary, price informativeness is measured by the conditional precision of the fundamental asset value, given the market price:

$$
\operatorname{var}\left(X \mid P_{\beta}\right)^{-1}=1+\frac{1}{\sigma_{z}^{2}}\left(\frac{a_{\beta}}{d_{\beta}}\right)^{2} ; \quad \beta \in(0,1] .
$$

As long as the constraint $\lambda_{1} \leq 1$ does not bind, Theorem 1 establishes that $\operatorname{var}\left(X \mid P_{\beta}\right)=C \sigma_{\epsilon}^{2}$ for both the systematic and diversifiable risk models. This implies that comparative statics on price informativeness in the two models yield identical results. ${ }^{14}$ The trade-off between becoming informed or staying uninformed coincides in both classes of models, so price revelation depends on the same parameters as long as we have interior solutions.

On the other hand, the coefficients $d_{\beta}$, which measure the risk premium demanded by agents for a given supply shock $Z_{\beta}$, have different functional forms in the systematic and diversifiable risk models. Let us first consider what would happen in the two economies if no agent acquired information. In the systematic risk model where per-capita supply of noise is non-trivial agents will demand a premium $d_{1} Z_{1}$ for holding the risky asset. On the other hand,

\footnotetext{
${ }^{14}$ From the results of subsection 5.2 it follows that this result critically depends on the assumption of agents' homogeneity and fails to hold, for example, when agents have heterogeneous risk aversion coefficients.
} 
in the diversifiable-risk model per-capita noise is negligible and the price of the risky asset would simply be zero (i.e. equal to its unconditional expected value), since no risk premium would be demanded by agents in equilibrium. Once it becomes possible to acquire information, the equilibrium price in the systematic-risk model would include a new term that would depend on the final payoff of the risky asset, thereby making the asset price partially revealing (and at the same time changing the risk premium demanded by agents). The diversifiable-risk model would exhibit an even more dramatic change: the price of the risky asset would change from zero to a non-trivial random variable. In that case a new term proportional to the final payoff $X$ would show up in the price function due to trading by the informed; in addition, a non-trivial risk-premium arises (the $d_{\beta} Z_{\beta}$ term). This allows us to conclude that asymmetric information considerations increase the volatility of the risky asset price. In the systematic risk model, on the other hand, the introduction of asymmetric information has an ambiguous sign: in some cases it increase and in others decrease price volatility. ${ }^{15}$ Along this dimension, therefore, the models behave quite differently.

Difference in the risk-premium coefficients $d_{\beta}$ drives a wedge between the two models and leads to some interesting comparative statics differences. In the systematic risk model, informed agents play a non-trivial role in terms of risk-sharing with respect to the whole investor population. Therefore both their risk-aversion and their conditional variance affect the riskpremium term $d_{1}$. When the supply risk is negligible on a per-capita basis, informed agents' risk-sharing capabilities are negligible compared to that of the uninformed. The equilibrium risk-premium is therefore affected by the informed agents only to the extent that their trades affect the information revealed by prices. To see the effects of this difference, consider the unconditional volatility in both models. In the diversifiable-risk model it is straightforward to check that $\operatorname{var}\left(P_{\beta}\right)=1-C \sigma_{\epsilon}^{2}$, for all $0<\beta<1$. In this case, unconditional volatility has simple comparative statics with respect to the model's primitives: it is decreasing in $\sigma_{\epsilon}^{2}, c$ and $\tau$, and is completely independent of $\sigma_{z}^{2}$. It is worth remarking that these comparative statics do not hold in the systematic risk model, where it is possible for the unconditional asset price volatility to be either increasing or decreasing in these variables depending on the values of the primitives of the model. ${ }^{16}$ In addition, the difference in $d_{\beta}$ values for the two models yields distinct comparative statics for welfare measures and trading volume which we, for brevity, do not report. Thus, despite some similarities, the two classes of symmetric models have very

\footnotetext{
${ }^{15}$ To see that, consider the systematic risk model with parameter values equal to $\sigma_{\epsilon}^{2}=\sigma_{z}^{2}=1$, and $c=0.25$. It is easy to check that if $\tau=1$ introducing asymmetric information would decrease price volatility, whereas if $\tau=3 / 4$ introducing asymmetric information would increase it.

${ }^{16}$ One can easily verify that in the systematic risk model
}

$$
\operatorname{var}\left(P_{1}\right)=\frac{\sigma_{z}^{2} \operatorname{var}\left(X \mid P_{1}\right)^{-1}}{\left(\lambda_{1} r_{1}+\frac{1}{\tau}\left(1+\frac{\lambda_{1} r_{1}}{\tau \sigma_{z}^{2}}\right)^{-1}\right)^{2}} .
$$


different comparative statics.

\section{Applications}

In this section we apply the results of the previous section to analyze direct sales of information by an information monopolist, and study information acquisition in multi-asset markets and derivatives markets under conditions of asymmetric information. We find that despite some similarities, the systematic and diversifiable risk models lead, often, to very different predictions.

\subsection{Markets for information}

Our discussion in this subsection closely follows Admati and Pfleiderer (1986). We consider the problem facing a monopolist seller of information, who has perfect information about the value of the risky asset and considers selling her information directly, e.g. through a newsletter. The assumption of perfect information implies that the information seller would add noise to her signal before selling it, since otherwise price would fully reveal her information. We assume that there are $N$ agents who consider purchasing the signal, with the set of economic primitives as described in section 2. As before, we differentiate among large noise economies by studying the systematic and diversifiable risk models, and compare the optimal strategy of a monopolist seller of information in the two models.

We focus on the case where noise that the monopolist adds to the signal, before selling it, is personalized. As Admati and Pfleiderer (1986) demonstrate in the systematic risk case, adding personalized noise is the optimal way of adding noise to the signal in such an economy. In particular, the monopolist sells signals $Y_{i}=X+\epsilon_{i}$ to $m_{N} \leq N$ agents, where $\epsilon_{i}$ are i.i.d. random variables with precision $s_{N}$. The monopolist seller can choose both the number of agents he would like to serve, $m_{N}$, and the precision of the signal he offers, $s_{N}$ (both of these quantities, in general, depend on the number of agents in the economy, $N$ ). We restrict the precision of noise to be bounded from below by a positive constant $\ell$. The economic motivation for the introduction of the lower bound on precision is that the seller of information cannot reasonably expect to sell information without any content.

From the model setup it is clear that, after the information sales stage of the game has been completed, the rational expectations equilibrium that arises coincides with the one described in section 2. The interesting question is what happens at the information sales stage. Following Admati and Pfleiderer (1986), for a finite $N$, the problem that the information seller faces at 
that stage reduces to

$$
\max _{m_{N} \leq N, s_{N} \geq \ell} m_{N} \log \left(\frac{\operatorname{var}\left(X \mid Y_{i}, P_{N}\right)^{-1}}{\operatorname{var}\left(X \mid P_{N}\right)^{-1}}\right) ;
$$

where the conditional variances in (12) depend both on $s_{N}$ and $m_{N}$, and are stated explicitly in Lemma 0 in the Appendix. ${ }^{17}$ Given the model specification, i.e. the choice of $\beta$, the equilibrium size of the market for information and the precision of the signals may vary. We denote them as $m_{N}(\beta)$ and $s_{N}(\beta)$, respectively, and study their limiting behavior as $N \uparrow \infty$. Let $s_{\beta}=\lim _{N \rightarrow \infty} s_{N}(\beta)$.

The following proposition extends the results of Admati and Pfleiderer (1986) to the diversifiable risk case.

Proposition 1. The optimal information sales for the monopolist satisfy (8). Moreover:

(i) In the systematic risk model the monopolist serves all agents, $\lambda_{1}=1$, and adds a finite amount of noise $s_{1}=\max \left(\ell, 1 /\left(\tau \sigma_{z}\right)\right)$.

(ii) In the diversifiable risk model the monopolist sets $s_{\beta}=\ell$, and the information sales satisfy

$$
\lambda_{\beta}=\arg \max _{\lambda} \lambda \log \left(\frac{1+\ell+\lambda^{2} \ell^{2} /\left(\tau \sigma_{z}\right)^{2}}{1+\lambda^{2} \ell^{2} /\left(\tau \sigma_{z}\right)^{2}}\right) .
$$

Intuitively, the monopolist is facing a trade-off between, on one hand, selling to as many agents as possible while, on the other, controlling the information that is revealed by the price. In the systematic risk model, as discussed in Admati and Pfleiderer (1986), the information seller extracts surplus from all agents in the economy by serving everyone, and controls the damaging effects of price informativeness by adding non-trivial amount of noise to the signal that she sells. The nature of the solution in the diversifiable risk model is substantially different. If noise is diversifiable the monopolist cannot sell to the whole trader population: given any signal with bounded precision prices will become fully revealing in the large $N$ limit. Therefore, an interior solution for the size of the investor population being served arises (measured by parameter $\lambda_{\beta}$ ); in addition, the information seller controls the information revelation through prices by electing the precision of the signal to be as low as possible. ${ }^{18}$ For completeness of the discussion, note that the limiting equilibrium asset prices in both cases satisfy the linear functional form (6). However, pricing coefficients differ in the two models leading to different

\footnotetext{
${ }^{17}$ In a finite-agent economy, when solving (12) one would face an integer programming problem. Since we are solely interested in the limiting economies the issue can be ignored, however, because it leads to a negligible approximation error.

${ }^{18}$ If the lower bound on the signal precision did not exist, the monopolist would like to design a pricing scheme that would satisfy $\lim _{N} s_{N}(\beta)=0$ when $\beta \in(0,1)$. In this way, she would control the price revelation through the added signal error noise, and still sell to the whole investor population. However, it is not clear how such limiting information sales would be implemented.
} 
predictions with respect to price informativeness of trades as well as the expected trading volume.

\subsection{Information acquisition in multi-asset markets}

Up to now, we studied the economy with only one risky asset. Now, analyze what happens to the original asset price and the incentives to gather information when a second risky asset is introduced. For definiteness, we assume that initially there was a systematic risk asset in the economy and consider how the limiting equilibrium changes if we introduce another systematic risk asset (this extends the analysis of Admati (1985)). We, then, compare such limiting economy with the case when a diversifiable risk asset is introduced instead. This allows to extend our main result, Theorem 1, to the case when there are several risky assets in the economy.

Let us introduce a vector of payoffs $\mathbf{X}=\left(X_{a}, X_{b}\right)$, a two-dimensional normally distributed random variable with variances set equal to one and the correlation coefficient $\rho$; we use index $j=a, b$ to enumerate the risky assets. Unless otherwise specifically stated, all other assumptions and conventions of section 2 apply. Recall that there are $N$ agents in the economy with CARA preferences and risk aversion parameter $\tau$. At the information acquisition stage, an agent $i$ can choose to stay uninformed, purchase a signal $Y_{i j}=X_{j}+\epsilon_{i j}$ on one of the assets $j=a, b$ only (at a cost $c_{j}$ ), or purchase signals on both assets (at a cost $c_{d}=(1-\delta)\left(c_{a}+c_{b}\right)$, for some $\delta \geq 0$; note that we allow here for possible economies of scale). Here, $\epsilon_{i j}$ are i.i.d. random variables such that $\operatorname{var}\left(\epsilon_{i j}\right)=\sigma_{\epsilon}^{2}$. We introduce an augmented index $t=a, b, d$ to enumerate three informed agent "types" that can emerge at the information acquisition stage: index $a$ corresponds to agents who purchase only a signal on asset $a$, index $b$ for those that become informed only on asset $b$, and index $d$ for agents who buy signals on both assets. We will refer to agents of types $a$ or $b$ as "specialist," whereas agents of type $d$ will be called "generalists." Finally, we introduce $C_{t}=e^{2 \tau c_{t}}-1$, for $t=a, b, d$.

Each of the assets $j=a, b$ is subject to an aggregate supply shock $Z_{N j}$. Generalizing the one-asset case definitions, let $Z_{\beta j}=\lim _{N \rightarrow \infty} N^{-\beta_{j}} m_{N j}(\beta)$, for some $\beta_{j} \in(0,1], j=a, b$. Also define $\mathbf{Z}_{\beta}=\left(Z_{\beta_{a}}, Z_{\beta_{b}}\right)$. As in the economy with one risky asset, an asset $j$ can have diversifiable endowment risk, $\beta_{j} \in(0,1)$, or systematic endowment risk (when $\beta_{j}=1$ ). We further assume that $\operatorname{var}\left(\mathbf{Z}_{\beta}\right)=\sigma_{z}^{2} \mathbf{I}$, where $\mathbf{I}$ denotes the two by two identity matrix. While we explicitely describe only a symmetric economy, where assets only differ with respect to the endowment risk nature (diversifiable versus systematic), the assumptions that we make are purely for notational convenience and brevity, and do not affect the fundamental conclusions we draw from the model.

We denote finite-agent economy as $\mathcal{E}_{N}(\beta)$ where vector $\beta=\left(\beta_{a}, \beta_{b}\right)$ specifies the endowment 
risk properties of the economy. In what follows we focus on two particular models: $\beta=(1,0.5)$, which we refer to as a mixed risk model; ${ }^{19}$ and $\beta=(1,1)$, which we refer to as a systematic risk model and which serves as our benchmark. In both of these models asset $a$ has systematic endowment risk. The difference between the models stems from the asset $b$ : in the mixed risk model that asset has diversifiable risk whereas in the systematic risk model that asset is another systematic risk asset. ${ }^{20}$

We now turn to characterize the equilibrium properties of an economy $\mathcal{E}_{N}(\beta)$ when the number of agents $N$ grows without bound. As in previous sections, we let $m_{N t}(\beta)$ and $\mathbf{P}_{N}(\beta)$ denote the endogenously determined number of traders of type $t$, and the equilibrium price vector of the risky assets respectively. Let us define

$$
\begin{aligned}
& \lambda_{a}(\beta)=\lim _{N \rightarrow \infty} N^{-\beta_{a}} m_{N a}(\beta) ; \\
& \lambda_{b}(\beta)=\lim _{N \rightarrow \infty} N^{-\beta_{b}} m_{N b}(\beta) ; \\
& \lambda_{d}(\beta)=\lim _{N \rightarrow \infty} N^{-\beta_{b}} m_{N d}(\beta) .
\end{aligned}
$$

The next proposition generalizes our previous results to this multi-asset setting.

Proposition 2. Consider a sequence of economies $\mathcal{E}_{N}(\beta)$. The equilibrium price vector satisfies:

$$
\mathbf{P}_{\beta} \equiv \lim _{N \uparrow \infty} \mathbf{P}_{N}(\beta)=\mathbf{A}_{\beta} \mathbf{X}-\mathbf{D}_{\beta} \mathbf{Z}_{\beta}
$$

for some matrices $\mathbf{A}_{\beta}, \mathbf{D}_{\beta} \in \mathbb{R}^{2 \times 2}$.

If the parameter values of the model are such that $\mathbf{D}_{\beta}^{-1} \mathbf{A}_{\beta}$ has full rank, and if $\lambda_{d}(\beta)>0$, then the following results hold a.s..$^{21}$

(i) In the systematic risk model, $\beta=(1,1)$, equilibrium number of informed traders satisfies $\lambda_{a}(\beta)=\lambda_{b}(\beta)=0$. Moreover,

$$
\lambda_{d}(\beta)=\tau \sigma_{z} \sigma_{\epsilon}^{2} \sqrt{\frac{(1+\sqrt{1+\omega})}{\sigma_{\epsilon}^{2} C_{d}}-\frac{1}{1-\rho^{2}}} ;
$$

where $\omega=C_{d}\left(1+\left(\rho \sigma_{\epsilon}^{2}\right)^{2} C_{d} /\left(1-\rho^{2}\right)^{2}\right)$.

\footnotetext{
${ }^{19}$ As before, this mixed model is equivalent to any of the form $\beta=(1, \beta)$ for any $\beta \in(0,1)$.

${ }^{20}$ Our benchmark model $\beta=(1,1)$ model is a special case of Admati (1985). In contrast to that work, agents here are allowed to endogenously make information acquisition decisions. To the best of our knowledge no paper has attempted to analyze information acquisition activities in this type of multi-asset model before. Admati and Pfleiderer (1987) study the viability of allocations of information. As shown in their paper, the i.i.d. structure on the signals makes the viability question trivial: one can always find $\operatorname{costs} c_{i}$ such that any allocation of information, within the class of economies we study, is viable. Our analysis contributes to the literature on multi-asset markets by studying a particular class of information acquisition technologies explicitely.

${ }^{21}$ That is, with the exception of a subset of the parameter values with zero measure.
} 
(ii) In the mixed risk model, $\beta=(1,0.5), \lambda_{a}(\beta)>0$, and $\lambda_{b}(\beta)=0$. Moreover, letting $\phi$ denote the solution to

$$
\phi^{2}\left(C_{d}-C_{a}\right)-\frac{\left(1+C_{a}\right)}{\sigma_{\epsilon}^{2}} \phi-\frac{\rho^{2}}{C_{a}\left(1-\rho^{2}\right)^{2}}=0
$$

we have that

$$
\begin{aligned}
& \lambda_{a}(\beta)=\tau \sigma_{z} \sigma_{\epsilon}^{2} \sqrt{\frac{\rho^{2}}{\phi\left(1-\rho^{2}\right)^{2}}+\frac{1}{C_{a} \sigma_{\epsilon}^{2}}-\frac{1}{1-\rho^{2}}} \\
& \lambda_{d}(\beta)=\tau \sigma_{z} \sigma_{\epsilon}^{2} \sqrt{\phi-\frac{1}{1-\rho^{2}}}
\end{aligned}
$$

The proposition first establishes an analogous convergence result to that in the single asset case of Theorem 1: prices converge to a limiting random variable that is independent of individual agents' signals, and solely depends on the asset payoff vector $\mathbf{X}$ and the noise term $\mathbf{Z}_{\beta}$. Moreover, when prices satisfy a natural rank condition, namely that agents learn from both prices, the proposition gives precise formulae for the amount of informed trading when the signals are complements, i.e. when there is a strict subset of the population that becomes informed. ${ }^{22}$

In the systematic risk model the proposition establishes that a fraction of agents becomes generalist, there would be no agents who would become specialists in either of the assets. This is rather intuitive given the symmetry of the model. In particular, it is straightforward to see that under our symmetry assumptions either all agents become specialists, or all agents become generalist.

In the mixed risk model, the asset with systematic risk has a full-measure of informed agents trading on the basis of private information, whereas for the asset with diversifiable risk only a small group of agents (in per capita terms) gather information. Moreover, it is immediate that the presence of the second asset, even though it is "small," affects the equilibrium price, and the amount of information gathered, in the first asset. Rather intuitively, since the small asset's price reveals information, the large asset market clearing condition is affected, as so will be both the properties of the large asset price (trading volume, volatility), as well as the incentives to gather information on this asset. Finally, note that the existence of generalists does not rule out the presence of specialists, in contrast to the systematic risk model.

To illustrate the perverse effect that the introduction of the diversifiable risk asset $b$ has on asset $a$, consider the following parameter values: $c_{a}=c_{b}=0.3, \delta=0.2$, and $\sigma_{\epsilon}^{2}=\tau=1$. The top panel of Figure 1 plots the equilibrium fraction of asset $a$ specialists in the mixed model

\footnotetext{
${ }^{22}$ This is precisely the case where the mixed and systematic risk models differ most clearly. See the proof of the proposition for details on how to compute the equilibria in other cases.
} 
(solid line), as well as in the one-asset economy (dashed line), as a function of the correlation between the two assets. The bottom panel plots the equilibrium values for the measure of generalists in the mixed model (solid line). ${ }^{23}$ Rather intuitively, as the correlation between the assets goes up informed trading goes down. Moreover, the fraction of asset $a$ specialists in the mixed model tends to zero, i.e. the only equilibria that survive as the correlation goes up is one in which a small subset of the population is a generalist, and no agent becomes a specialist on asset $a .^{24}$ This highlights the fact that information revelation across asset markets can make assets with diversifiable risk play a bigger role than one would expect. The lower panel of Figure 1 also plots the equilibrium fraction of informed agents in the systematic risk model (dotted line). In contrast to the mixed risk model, for high values of correlation there is always an interior fraction of generalists in the equilibrium in information acquisition (and no specialists).

The proposition also establishes that there will always be a fraction of agents who buy information on asset $a$, which in the systematic risk model never occurs. In general, under the rank condition of the proposition, the mixed risk model has two separate markets: one for agents trading on the basis of signals on asset $a$, and one in which agents possess information on asset $b$ (and maybe also asset $a$ ). The key distinction is that in the diversifiable risk model the measure of generalists $\lambda_{d}(\beta)$, has no effect on the information revealed by the price of asset $a$. The intuition for this is that the noise of asset $a$ screens out the informed trades of the generalists, since the equilibrium fraction of generalists tends to zero. On the other hand, in the systematic risk model the information acquisition decisions of agents who purchase both signals affects directly the information revealed by prices on both assets. Clearly, the incentives to gather extra information in the diversifiable risk model are different.

\subsection{Derivatives trading}

In this section we extend the work by Brennan and Cao (1996) and Cao (1999) to the diversifiable risk model. In particular, consider the setup introduced in section 2 , but let us introduce a security, which is in zero-net supply, whose payoff is $\left(X-P_{N}\right)^{2}$. This security will trade alongside the stock, and we let $Q_{N}$ denote its price in the $N$ agent economy. Agents now choose their trading strategies in the stock market, which we denote by $\theta_{i}$, and in the derivatives market, where $\gamma_{i}$ denotes the number of units of the quadratic security agent $i$ purchases. Final wealth is therefore given by $W_{i}=\theta_{i}\left(X-P_{N}\right)+\gamma_{i}\left(\left(X-P_{N}\right)^{2}-Q_{N}\right)$. The rational expectations equilibrium is defined as in section 2 , with the additional requirement that the market for the quadratic derivative clears. Intuitively, this quadratic security will be traded in equilibrium as long as agents have different beliefs in terms of the conditional

\footnotetext{
${ }^{23}$ Note that $\lambda_{d}(\beta)$ can be greater than 1 in the mixed model, since it is not a fraction.

${ }^{24}$ We should remark that the model is symmetric in the correlation variable $\rho$.
} 
variances of the underlying asset. ${ }^{25}$ Informed agents will sell these securities short, whereas uninformed agents will buy them. The rest of the notation and assumptions is as in section 2 .

The next proposition studies the limiting rational expectations equilibrium when this quadratic derivative is available for trade.

Proposition 3. Consider a sequence of economies $\mathcal{E}_{N}(\beta)$, where $\beta \in(0,1]$, and agents can trade on quadratic derivatives. Limiting prices satisfy (6), where the price coefficients are as given in Theorem 1. Moreover, the limiting equilibrium satisfies (8) for some $\lambda_{\beta}>0$. In particular, the introduction of the derivative security will increase the amount of information acquisition.

(i) In the systematic risk model, $\beta=1$, the derivative price satisfies

$$
\lim _{N \rightarrow \infty} \frac{1}{Q_{N}}=\lambda_{1} \operatorname{var}\left(X \mid P_{1}, Y_{i}\right)^{-1}+\left(1-\lambda_{1}\right) \operatorname{var}\left(X \mid P_{1}\right)^{-1}
$$

(ii) In the diversifiable risk model, $\beta \in(0,1)$, limiting prices satisfy

$$
\lim _{N \rightarrow \infty} \frac{1}{Q_{N}}=\operatorname{var}\left(X \mid P_{\beta}\right)^{-1}
$$

Brennan and Cao (1996) and Cao (1999) developed this model in the standard systematic risk model. Absent information acquisition activities, the asset price coefficients are unaltered with respect to the model without a derivative asset. Moreover, the price of this derivative is given by a weighted average of the conditional precisions of informed and uninformed, as (21) shows. Cao (1999) further established that the derivative asset will induce more information acquisition activities.

In the diversifiable risk model the informed agents are not marginal buyers/sellers in the derivatives market: even though they do take non-trivial positions (they are short the quadratic asset), the price of the derivative is determined solely by the uninformed, since their riskbearing capacity is an order of magnitude larger. This implies the conditional variance of $X$ given $P_{N}$ characterizes the limiting option prices. Further note that the option price is lower in the systematic risk case, since there the conditional precision of the informed also plays a non-trivial role in the quadratic derivative market: when there is systematic risk the informed agents will be marginal on the derivatives markets. These differences generate different implications in terms of trading volume on the options market, as well as in the stock

\footnotetext{
${ }^{25}$ These quadratic terms achieve Pareto optimal risk-sharing when CARA agents share risk with normally distributed payoffs with different beliefs on the mean and variance. They were already discussed by Wilson (1968), who labeled them "side bets."
} 
market (for the same reasons as in section 3.2). ${ }^{26}$ On the other hand, note that one of the main predictions of the model, that information acquisition activities are increased with the introduction of the derivative asset, is robust across the two models.

\section{$5 \quad$ Extensions}

We first study whether our results are dependent on the price taking assumption. In particular, we study a sequence of economies as in Kyle (1989), and show that their limits coincide with those present in Theorem 1. Next, we extend the results in section 3 to allow for heterogeneous risk-aversion. Finally, we analyze a model in which the aggregate supply of the risky asset stems from random observable shocks to individual agents, and show the equilibrium exhibits similar properties to those discussed in section 3 .

\subsection{Imperfect competition and competitive limits}

In this section we closely follow the analysis of rational expectations equilibria with imperfect competition in Kyle (1989). The finite agent economy has prices which are conjectured to be of the form (3). ${ }^{27}$ In contrast to our previous analysis, agents take into account that their trades affect prices. In particular, each agent $i$ conjectures that she faces a residual supply curve of the form $P_{N}\left(\theta_{i}\right)=P_{N i}+d_{N i} \theta_{i}$, for some intercept $P_{N i}$ and slope $d_{N i}>0 .{ }^{28}$ The rest of the elements of the model are as described in section 2. An economy is described by a total number $N$ of CARA traders with the risk-aversion parameter $\tau$, who can obtain information for a cost $c$ with signal error variance $\sigma_{\epsilon}^{2}$, and by aggregate noise summarized by the parameters $\beta$ and $\sigma_{z}^{2}$

Fixing the number of informed agents $m$ and the total number of traders $N$, a rational expectations equilibrium is defined by a set of trading strategies $\theta_{i}$ that solve

$$
\theta_{i} \in \arg \max _{\theta} \mathbb{E}\left[u\left(\theta_{i}\left(X-P_{N}\left(\theta_{i}\right)\right)\right) \mid \mathcal{F}_{i}\right] ; \quad i=1, \ldots, N
$$

and a price function of the form (3) such that the market clearing condition (2) holds.

At the information acquisition stage we proceed as in the previous analysis, by equating the ex-ante expected utilities of informed and uninformed. As before, this yields an endogenously

\footnotetext{
${ }^{26}$ One can also verify, using the expressions in the proof, that the coefficients $\lambda_{\beta}$ are different in the two models.

${ }^{27}$ In contrast to indivisible unit auctions, in this type of auctions the bidders are allowed to bid fractional amounts of good. See, for example, Wilson (1979), Back and Zender (1993) and Viswanathan, Wang, and Witelski (2001).

${ }^{28}$ Note that this conjecture will be verified in equilibrium. See Kyle (1989) for details on the rational expectations equilibrium definition under imperfect competition.
} 
determined number of informed traders $m_{N}(\beta)$, whose behavior for large $N$, as a function of the type of economy (diversifiable or systematic), is the main object of study. We should remark that this is the main departure point from the discussion of large markets in Kyle (1989): as noise grows we endogenize the information acquisition decisions, and in consequence the allocation of private signals in the economy.

The next proposition shows that the limiting equilibria coincide with those in Theorem 1.

Proposition 4. The economy with imperfect competition with endogenous information acquisition exhibits the same limiting prices, limiting optimal trading strategies, and limiting measures of informed trading as the competitive model in Theorem 1, both in the systematic and the diversifiable risk models.

The intuition for the proposition is fairly straightforward given the limiting results in Kyle (1989). ${ }^{29}$ In essence, in large economies with growing noise, either systematic or diversifiable, agents' strategic decisions become irrelevant. Since trading behavior is non-strategic, the limiting equilibria converges to the perfectly competitive one. It is well know that the Kyle (1989) model can be viewed as a share auction. ${ }^{30}$ Albeit in a very stylized type of auction setting, the proposition shows that the intuition from Swinkels (1999) and Swinkels (2001) extends well beyond the private values case: large noise eliminates strategic behavior. This result thus gives the limiting competitive economies described in section 3 a wider applicability: for a large class of strategic models the limiting equilibrium is indeed competitive, and given by the expressions in Theorem 1 .

The auction literature, when studying information aggregation, has typically focused on perfect revelation of information in markets with large numbers of risk-neutral bidders. ${ }^{31}$ As discussed in Jackson (2003), this yields a Grossman and Stiglitz (1980) type of impossibility result if information is costly to acquire. The introduction of noise, typical in rational expectations models, is a mechanism that can prevent auction prices from perfectly revealing information. Proposition 4 shows one particular auction setting in which prices aggregate information in a natural way, and information acquisition activities are derived endogenously. Moreover, it provides a set of necessary and sufficient conditions on noise for which such limiting equilibria exist. There are some interesting analogies between the size of aggregate supply we discuss in this paper, and the literature in multi-unit auctions with large number of agents. ${ }^{32}$ In particular, the diversifiable risk model has a similar flavor to auction models where the fraction of agents who receive a good goes to zero as the number of agents increases;

\footnotetext{
${ }^{29}$ But note that, although the flavor is similar, the result is not a special case of the limits considered in Kyle (1989), neither for the systematic or diversifiable risk models.

${ }^{30}$ See for example Brunnermeier (2001).

${ }^{31}$ See Wilson (1977), Milgrom (1979), Milgrom (1981), Pesendorfer and Swinkels (1997), Pesendorfer and Swinkels (2000), and Kremer (2002).

${ }^{32}$ See, for example, Swinkels (2001), Jackson (2003), Jackson and Kremer (2004), Hong and Shum (2004).
} 
whereas the systematic risk model can be compared to an auction model where a fraction of the agents receive the good in the limiting economies. Whether the types of noise introduced in this paper may have similar effects in other auction settings seems like an interesting research route.

\subsection{Heterogeneous risk-aversion}

We consider next the effects of heterogeneity in the risk aversion of individual agents, fixing the information acquisition technology. The definition of a rational expectations equilibrium is identical as in section 2. Now we allow the $N$ agents to have $K$ different risk-aversion parameters, which we denote by $\tau_{k}$, for $k=1, \ldots, K$. Let $\tau_{\min }=\min _{k}\left\{\tau_{k}\right\}$. Each agent takes an action from the set $\{A, N\}$, where $A$ denotes the action of purchasing, and $N$ denotes the action of not purchasing the signal.

A Nash equilibrium (in pure strategies) at the information acquisition stage is defined by two sets of agents, a set $\mathcal{I}$ (which denotes those agents that purchase signals), and a set $\mathcal{U}$ (which denotes those traders that remain uninformed), such that: (i) none of the agents in $\mathcal{U}$ desires to purchase a signal, and (ii) none of the agents in $\mathcal{I}$ desires not to purchase a signal, all taking as given that the other players follow their equilibrium strategies. ${ }^{33}$

For a finite $N$, multiple equilibria naturally arise. ${ }^{34}$ The next proposition characterizes the competitive limit in this economy.

Proposition 5. In the model with heterogeneous risk-aversion the only Nash equilibria that survives as $N$ grows large is the one where only agents with risk-aversion parameter $\tau_{\text {min }}$ become informed. The number of such informed traders satisfies (8), where $\lambda_{\beta}$ is given by

$$
\lambda_{\beta}=\tau_{\min } \sigma_{z} \sigma_{\epsilon} \sqrt{\frac{1}{C\left(\tau_{\min }\right)}-\sigma_{\epsilon}^{2}}
$$

where $C\left(\tau_{\min }\right)=e^{2 \tau_{\min } c}-1$.

The effects of heterogeneity go in the same direction as in Verrecchia (1982), namely the more risk-tolerant agents are more likely to become informed. But the situation in the diversifiable risk model is more extreme. In that case, only those agents with the lowest possible risk

\footnotetext{
${ }^{33}$ This is the standard equilibria in information acquisition discussed in most of the literature. Morrison and Vulkan (2003) studies the standard Kyle (1985) model with endogenous information acquisition, when traders face uncertainty with respect to the number of informed agents in the market at the time of trading. Due to the competitive nature of our model the issues that arise in Morrison and Vulkan (2003) with respect to off-equilibrium beliefs of informed traders do not arise here.

${ }^{34}$ It is easy to construct an example with two agents with different risk-aversion parameters, in which only one agent becomes informed in equilibrium, but it could be either of the two agents.
} 
aversion become informed, instead of a strictly positive subset of the type space. It is rather intuitive that more risk-tolerant agents will become informed: they trade more aggressively, thereby capturing more rents from their information acquisition activities. This standard intuition, together with the fact that the agents who become informed are negligible in size as $N \rightarrow \infty$, yields the conclusion of the proposition.

\subsection{Endowment risk}

Consider next a version of the model where noise stems from some random endowment shocks to each agent. In particular, prior to trading each agent observes his endowment of the risky asset, which we denote by $Z_{i}$. The $Z_{i}$ 's are assumed to be i.i.d. Gaussian random variables with zero mean and variance $\sigma_{z}^{2}$. Aggregate supply of the risky asset is then $Z_{N}=\sum_{i=1}^{N} Z_{i}$, and has a variance proportional to $N$ : this is effectively a model with diversifiable risk. ${ }^{35}$ Using the notation of the previous sections, the final wealth for agent $i$ is given by $W_{i}=\theta_{i}\left(X-P_{N}\right)+$ $P_{N} Z_{i}$. All agents are assumed to have CARA preferences with risk-aversion parameter $\tau$. The model described thus far is a simple generalization of Diamond and Verrecchia (1981), who only study the case $m_{N}=N$, i.e. the case where all agents are informed.

We will conjecture, as usual, that price is linear in the random variables $Y_{i}$ and $Z_{i}$. Now there is heterogeneity in the random supply: a portion will stem from informed agents, and a portion from the uninformed. Therefore, we will search for price functions of the form

$$
P_{N}=\sum_{i=1}^{m_{N}} b_{i} Y_{i}-\sum_{i=1}^{m_{N}} d_{i} Z_{i}-\sum_{i=m_{N}+1}^{N} d_{i} Z_{i} .
$$

At the ex-ante stage we proceed as before: we determine the equilibrium number of informed agents by equating the expected utilities of the informed and the uninformed. The next proposition shows that the limiting behavior of this economy is asymptotically identical to that in Theorem 1.

Proposition 6. The number of informed agents satisfies $\lim _{N \rightarrow \infty} m_{N} N^{-1 / 2}=\lambda_{0.5}$, where $\lambda_{0.5}$ is given in Theorem 1. The price function (24) converges to (6).

It should be noted that the models do differ in the finite-agent case: in the above model with endowment shocks even the uninformed agents have some private information, namely the realization of $Z_{i}$. Therefore their trading strategy is slightly more complicated than in the model with noise traders, although in the limit they converge. The above proposition

\footnotetext{
${ }^{35}$ Similar statements can be made about the equivalence that we will establish below for the systematic risk model.
} 
thereby links models with endowment risk and noise traders, by showing that limiting prices are identical under either interpretation of noise.

\section{Conclusion}

We have studied the role of noise in large competitive economies. The paper has uncovered a new limiting rational expectations equilibrium that possesses many of the nice properties of the standard model (e.g. partially revealing prices, closed-form solutions). We have shown that the new model, which is characterized by noise that is diversifiable, has a life of its own, since risk-sharing and price revelation play different roles than in the standard model. In general, these two models have different comparative statics implications with respect to price informativeness, price volatility, and other important economic characteristics. We have also shown that the limiting equilibria in such economies are perfectly competitive even when a strategic solution concept is used, thereby providing one more illustration of a model where perfect competition is the outcome of strategic trading among large number of agents. The new equilibria emphasizes the importance of information revelation in asset markets, and highlights the fact that small amounts of noise can support partially revealing equilibrium prices.

We demonstrated that differences in predictions between the systematic risk and diversifiable risk can be significant in applications. We established that a monopolist seller of information will optimally sell as imprecise a signal as possible when facing diversifiable risk, in sharp contrast to the systematic risk model. It has also been shown that incentives to invest in information acquisition in assets with systematic risk can be altered if a diversifiable asset becomes available for trade, due to information revelation across markets. Moreover, when there are complementarities in information acquisition activities, the model with a diversifiable asset differs with respect to a multi-asset model with only systematic risk assets. Lastly, our analysis of derivative markets highlights the main property of the new equilibria, that informed agents have negligible risk sharing capacity vis a vis the uninformed, and therefore cannot be marginal price setters in option markets. Nonetheless, they will impact the equilibria through the information revealed by price. To what extent the diversifiable versus systematic risk models have different implications in other areas of applied research does seem an interesting route for future work, given our results on these three problems. 


\section{Appendix}

We commence by stating a lemma which we will use throughout the proofs. The next lemma solves for the equilibrium price in the finite economy $\mathcal{E}_{N}(\beta)$ in closed-form, and characterizes the endogenously determined number of informed traders. We let $n_{N}$ denote the number of uninformed agents, where $n_{N}=N-m_{N}$. Moreover, let $V_{z}^{2}=\operatorname{var}\left(Z_{N}\right)$ denote the variance of aggregate supply. Finally, in a slight abuse of notation, we use $\mathcal{F}_{I}$ and $\mathcal{F}_{U}$ to denote the information sets of typical informed and uninformed agents, respectively. We drop the subscript $N$ in the statement of the proposition for notational clarity (all price coefficients, as well as $m$ and $V_{z}$, depend on $N$ ). The proof of the lemma is omitted, and follows from the expressions in Hellwig (1980) and Admati and Pfleiderer (1987).

Lemma 0 (Finite-agent economy equilibrium). Let $m \geq 2$. Then, there exists a symmetric equilibrium of the form (3), in which ${ }^{36}$

$$
d=\frac{d_{u}}{d_{l}} ; \quad \frac{b}{d}=r
$$

where $r$ is the solution to

$$
r^{3}+r \frac{V_{z}^{2}}{(m-1) \sigma_{\epsilon}^{2}}-\frac{V_{z}^{2}}{\tau(m-1) \sigma_{\epsilon}^{4}}=0 .
$$

and

$$
\begin{gathered}
d_{u}=1+\frac{n m r}{\tau\left(m r^{2} \sigma_{\epsilon}^{2}+V_{z}^{2}\right)}+\frac{m(m-1) r}{\tau\left(V_{z}^{2}+r^{2} \sigma_{\epsilon}^{2}(m-1)\right)} \\
d_{l}=\frac{n\left(r^{2} m\left(m+\sigma_{\epsilon}^{2}\right)+V_{z}^{2}\right)}{\tau\left(m r^{2} \sigma_{\epsilon}^{2}+V_{z}^{2}\right)}+\frac{m\left(V_{z}^{2}\left(\sigma_{\epsilon}^{2}+\sigma_{x}^{2}\right)+\sigma_{\epsilon}^{2} r^{2}(m-1)\left(m+\sigma_{\epsilon}^{2}\right)\right)}{\tau \sigma_{\epsilon}^{2}\left(V_{z}^{2}+r^{2} \sigma_{\epsilon}^{2}(m-1)\right)}
\end{gathered}
$$

The equilibrium number of informed traders, ignoring integer constraints, is given by the condition

$$
\frac{\operatorname{var}\left(X \mid \mathcal{F}_{U}\right)}{\operatorname{var}\left(X \mid \mathcal{F}_{I}\right)}=e^{2 \tau c}
$$

where

$$
\begin{gathered}
\operatorname{var}\left(X \mid \mathcal{F}_{U}\right)=\left(1+\frac{r^{2} m^{2}}{m r^{2} \sigma_{\epsilon}^{2}+V_{z}^{2}}\right)^{-1} ; \\
\operatorname{var}\left(X \mid \mathcal{F}_{I}\right)=\left(1+\frac{1}{\sigma_{\epsilon}^{2}}+\frac{r^{2}(m-1)^{2}}{r^{2}(m-1) \sigma_{\epsilon}^{2}+V_{z}^{2}}\right)^{-1} .
\end{gathered}
$$

\footnotetext{
${ }^{36}$ Note that the condition $m=2$ is innocuous, since we will mostly be interested in interior solutions, i.e. markets where as $N \uparrow \infty$ the number of informed traders will be some positive amount, and ignore for the most part corner solutions.
} 


\section{Proof of Lemma 1.}

The price function can be expressed as

$$
P_{N}=d_{N} N^{\beta}\left[m_{N} N^{-\beta} r_{N}\left(X+\bar{e}_{N}\right)-Z_{\beta N}\right]
$$

where $Z_{\beta N} \equiv N^{-\beta} Z_{N}, \bar{e}_{N}=\left(m_{N}\right)^{-1} \sum_{i=1}^{m_{N}} \epsilon_{i}$, and $r_{N}=b_{N} / d_{N}$. Note that $\hat{Z}_{\beta N}$ has a nondegenerate limiting distribution by assumption.

First we show sufficiency. If $\alpha=\beta$, then from (26), and using our conjecture (5), we see that $\lim _{N \rightarrow \infty} r_{N}=\frac{1}{\tau \sigma_{\epsilon}^{2}}>0$. From this equation it is immediate that the limiting prices are partially revealing as long as $\beta>0$. Also note that (6) follows from the strong law of large numbers.

We show necessity by contradiction. First suppose that $\alpha<\beta$. Then (26) yields $\lim _{N \rightarrow \infty} r_{N}=$ $\frac{1}{\tau \sigma_{\epsilon}^{2}}>0$. From (30) it is immediate that the limiting prices are completely uninformative about $X$. Now suppose that $\alpha>\beta$. If $\beta \geq \alpha / 2$ we again have that $\lim _{N \rightarrow \infty} r_{N}>0$, and by inspection of (30) we see that prices become fully revealing as $N \rightarrow \infty$. If $\beta<\alpha / 2$, first note that, letting $\gamma=2 \beta-\alpha<0$, from (26) we have $r_{N}=\left(\frac{N^{\gamma} \sigma_{z}^{2}}{\tau \lambda_{\alpha} \sigma_{\epsilon}^{4}}\right)^{1 / 3} \xi(N)$, where $\lim _{N \rightarrow \infty} \xi(N)=1$, or $r_{N} \approx N^{\gamma / 3} c$, for some constant $c>0$. The coefficient that multiplies $X$ in (30) is therefore approximately of the order $N^{(2 \alpha-\beta) / 3}$, so that prices become fully revealing. This shows that $\alpha=\beta$ is a necessary condition for prices to be asymptotically partially revealing.

\section{Proof of Theorem 1.}

We start by conjecturing that the number of informed agents satisfies (5). If $\alpha<\beta$, taking limits in $(27)$ we have

$$
\lim _{N \rightarrow \infty} \frac{\operatorname{var}\left(X \mid \mathcal{F}_{U}\right)}{\operatorname{var}\left(X \mid \mathcal{F}_{I}\right)}=1+\frac{1}{\sigma_{\epsilon}^{2}} ;
$$

and this limiting value is larger than $e^{2 \tau c}$ by assumption. Therefore $\alpha<\beta$ cannot characterize a limiting equilibrium with endogenous information gathering. On the other hand, if $\alpha>\beta$ then

$$
\lim _{N \rightarrow \infty} \frac{\operatorname{var}\left(X \mid \mathcal{F}_{U}\right)}{\operatorname{var}\left(X \mid \mathcal{F}_{I}\right)}=1
$$

which obviously cannot be compatible with a limiting equilibrium with information acquisition. This argument implies that in any limiting equilibrium with endogenous information acquisition decisions (8) must hold.

In order to compute the equilibrium measures of informed agents we note that for $\alpha=\beta$ 
we have

$$
\lim _{N \rightarrow \infty} \frac{\operatorname{var}\left(X \mid \mathcal{F}_{U}\right)}{\operatorname{var}\left(X \mid \mathcal{F}_{I}\right)}=\frac{1+\frac{1}{\sigma_{\epsilon}^{2}}+\frac{1}{\sigma_{z}^{2}}\left(\frac{\lambda_{\beta} b_{\beta}}{d_{\beta}}\right)^{2}}{1+\frac{1}{\sigma_{z}^{2}}\left(\frac{\lambda_{\beta} b_{\beta}}{d_{\beta}}\right)^{2}}
$$

Using (27) we immediately arrive at the expressions for $\lambda_{\beta}$ given in the proposition. Finally, taking formal limits in the expressions from Lemma 0 yields the expressions for the price coefficients given in the Proposition. This concludes the proof.

\section{Proof of Proposition 1.}

By arguments similar to those in Theorem 1 we see that the monopolist seller of information optimal number of sales must satisfy (8), for some constant $\lambda_{\beta}$. One can easily verify that

$$
\begin{gathered}
\lim _{N \uparrow \infty} \operatorname{var}\left(X \mid \mathcal{F}_{I}\right)^{-1}=1+s_{\beta}+\left(\frac{\lambda_{\beta} s_{\beta}}{\tau}\right)^{2} \frac{1}{\sigma_{z}^{2}} ; \\
\lim _{N \uparrow \infty} \operatorname{var}\left(X \mid \mathcal{F}_{U}\right)^{-1}=1+\left(\frac{\lambda_{\beta} s_{\beta}}{\tau}\right)^{2} \frac{1}{\sigma_{z}^{2}} .
\end{gathered}
$$

From these and (12) it is immediate that the monopolist problem reduces to

$$
\max _{\lambda, s} \quad \lambda \log \left(\frac{1+s+\lambda^{2} s^{2} /\left(\tau \sigma_{z}\right)^{2}}{1+\lambda^{2} s^{2} /\left(\tau \sigma_{z}^{2}\right)}\right)
$$

with the added constraints $s \geq \ell$ (for both the systematic and diversifiable risk models), and $\lambda \leq 1$ (for the systematic risk model).

For a fixed $\lambda$, the optimal noise added is characterized by $s=\tau \sigma_{Z} / \lambda$. Some simple calculations show that $s \geq \ell$ must bind at the optimal solution in the diversifiable risk model, whereas in the systematic risk model $\lambda_{1} \leq 1$ will be the binding constraint. The statements in the proposition follow from these observations.

\section{Proof of Proposition 2.}

Prices, in the finite agent economy, are conjectured to be linear in the signals received by the agents:

$$
\mathbf{P}_{\mathbf{N}}=\sum_{i=1}^{m_{N}} \mathbf{B}_{\mathbf{i}} \mathbf{Y}_{\mathbf{i}}-\mathbf{D} \mathbf{Z}_{\mathbf{N}}
$$

where $m^{*}=m_{N a}+m_{N b}+m_{N d}$ denotes the total number of informed, $\mathbf{Z}_{\mathbf{N}}=\left(Z_{N a}, Z_{N b}\right)$ is the vector of aggregate supplies, and $\mathbf{B}_{\mathbf{i}} \in \mathbb{R}^{2 \times 2}$ and $\mathbf{D} \in \mathbb{R}^{2 \times 2}$ are the equilibrium price coefficients. The ex-ante utility (gross of information costs) of an agent whose information at the time of trading is given by the filtration $\mathcal{F}_{i}$ is $-\left|\operatorname{var}\left(\mathbf{X} \mid \mathcal{F}_{i}\right)\right|^{-1 / 2}$. The (endogenously) 
number of informed traders of each of the types is given by the natural indifference conditions (and corresponding inequalities), as in (27).

By similar arguments to those in the proof of Theorem 1 we have that (14)-(16) must have finite limits (possibly zero), otherwise prices will perfectly reveal information. Formally taking limits in (31), we arrive at an expression of the form (17), where in the mixed risk model

$$
\mathbf{D}_{\beta}^{-1} \mathbf{A}_{\beta}=\left[\begin{array}{cc}
\left(\frac{\lambda_{a}(\beta)}{\tau \sigma_{\epsilon a}^{2}}\right) & 0 \\
0 & \left(\frac{\lambda_{b}(\beta)+\lambda_{d}(\beta)}{\tau \sigma_{\epsilon b}^{2}}\right)
\end{array}\right]
$$

and in the systematic risk model

$$
\mathbf{D}_{\beta}^{-1} \mathbf{A}_{\beta}=\left[\begin{array}{cc}
\left(\frac{\lambda_{a}(\beta)+\lambda_{d}(\beta)}{\tau \sigma_{\epsilon a}^{2}}\right) & 0 \\
0 & \left(\frac{\lambda_{b}(\beta)+\lambda_{d}(\beta)}{\tau \sigma_{\epsilon b}^{2}}\right)
\end{array}\right]
$$

The following three conditions are the candidates for characterizing the equilibrium measures of informed trading $\lambda_{a}, \lambda_{b}$ and $\lambda_{d}$ in both models:

$$
\begin{aligned}
\kappa_{a}\left|\operatorname{var}\left(\mathbf{X} \mid \mathcal{F}_{a}\right)^{-1}\right| & =\left|\operatorname{var}\left(\mathbf{X} \mid \mathcal{F}_{U}\right)^{-1}\right| \\
\kappa_{b}\left|\operatorname{var}\left(\mathbf{X} \mid \mathcal{F}_{b}\right)^{-1}\right| & =\left|\operatorname{var}\left(\mathbf{X} \mid \mathcal{F}_{U}\right)^{-1}\right| \\
\kappa_{d}\left|\operatorname{var}\left(\mathbf{X} \mid \mathcal{F}_{d}\right)^{-1}\right| & =\left|\operatorname{var}\left(\mathbf{X} \mid \mathcal{F}_{U}\right)^{-1}\right|
\end{aligned}
$$

where $\mathcal{F}_{t}$ denotes the information possessed by an agent of type $t$, and $\kappa_{t} \equiv 1 /\left(1+C_{t}\right)$, for $t=a, b, d$. Equations (34)-(36) represent the set of indifference conditions for agents who purchase one signal on asset $a$, one signal on asset $b$, or signals on both assets.

Let $s_{\epsilon} \equiv 1 / \sigma_{\epsilon}^{2}$ denote the precision of the signal error. Define $\mathbf{H}=\mathbf{V}_{\mathbf{x}}^{-\mathbf{1}}$, and let $H_{i j}$ denote the $i j$ th component of the matrix $\mathbf{H}$, which we subscript using the asset indexes $a$ and $b$. Some simple manipulations yield that in the mixed risk model

$$
\operatorname{var}\left(\mathbf{X} \mid \mathbf{P}_{\beta}\right)^{-1}=\left[\begin{array}{cc}
x_{a} & -H_{a b} \\
-H_{a b} & x_{b}
\end{array}\right]
$$

where

$$
\begin{gathered}
x_{a} \equiv H_{a a}+\left(\frac{\lambda_{a} s_{\epsilon}}{\tau}\right)^{2} \frac{1}{\sigma_{z}^{2}} \\
x_{b} \equiv H_{b b}+\left(\frac{\left(\lambda_{b}+\lambda_{d}\right) s_{\epsilon}}{\tau}\right)^{2} \frac{1}{\sigma_{z}^{2}} .
\end{gathered}
$$


With this notation, the system (34)-(36) reduces to

$$
\begin{aligned}
\kappa_{a}\left(\left(x_{a}+s_{\epsilon}\right) x_{b}-\kappa_{0}\right) & =x_{a} x_{b}-\kappa_{0} ; \\
\kappa_{b}\left(x_{a}\left(x_{b}+s_{\epsilon}\right)-\kappa_{0}\right) & =x_{a} x_{b}-\kappa_{0} ; \\
\kappa_{d}\left(\left(x_{a}+s_{\epsilon}\right)\left(x_{b}+s_{\epsilon}\right)-\kappa_{0}\right) & =x_{a} x_{b}-\kappa_{0} ;
\end{aligned}
$$

where $\kappa_{0}=H_{a b}^{2}$.

By inspection, it is immediate that (40)-(42) cannot all be satisfied (with the exception of subsets of the parameter space of measure zero), and therefore at most two of those equations

will bind at the optimal solution. Moreover, when $\hat{\mathbf{D}}_{\beta}^{-1} \hat{\mathbf{A}}_{\beta}$ has full rank, it is immediate from (32) that in the diversifiable risk model (40) must bind. If $\lambda_{d}>0$, then (42) must also bind. The expressions in part (ii) of the proposition are the solutions to (40) and (42) expressed in terms of the $\lambda_{t}$ 's using (38) and (39).

In the systematic risk model, information acquisition decisions are characterized by the same system (40)-(42), where $x_{b}$ is also given by (39), but instead of (38) we have

$$
x_{a} \equiv H_{a a}+\left(\frac{\left(\lambda_{a}+\lambda_{d}\right) s_{\epsilon}}{\tau}\right)^{2} \frac{1}{\sigma_{z}^{2}} .
$$

The systematic risk model differs from the diversifiable risk model in this mapping from the $\lambda_{t}$ 's to the variables $x_{a}$ and $x_{b}$, and also on the set of constraints that bind. In particular, on top of the system (40)-(42), we need to restrict, for the obvious economic reasons, $\lambda_{t} \geq 0$, for $t=0, a, b, d$, and $\lambda_{a}+\lambda_{b}+\lambda_{d} \leq 1$. It is straightforward to check that if $\lambda_{d}>0$, so that (42) binds, then (40) or (41) cannot bind in the symmetric model. Note that in this case only one of the indifference conditions (40)-(42) binds. Some straightforward calculations yield the expression in part (i) of the proposition.

\section{Proof of Proposition 3.}

We consider a finite-agent version of the Brennan and Cao (1996), and explicitely take limits, as in previous proofs. First consider the finite $N$ model. It is straightforward to show that the optimal trade in the derivative security for agent $i$ are given by

$$
\gamma_{i}^{*}=\frac{1}{2 \tau}\left(\frac{1}{Q_{N}}-\frac{1}{\operatorname{var}\left(X \mid \mathcal{F}_{i}\right)}\right)
$$

One can easily check that in the finite-agent model, as in Brennan and Cao (1996), trades in the derivative security do not change the equilibrium price of the stock, which is given by 
the expressions in Lemma 0. Market clearing in the derivative asset immediately implies that

$$
\frac{1}{Q_{N}}=\frac{1}{N} \sum_{i=1}^{N} \operatorname{var}\left(X \mid \mathcal{F}_{i}\right)^{-1} .
$$

Calculating the ex-ante expected utilities in the presence of the derivative securities, and using the indifference condition which equates the expected utilities of informed and uninformed one arrives at the analog of (27) in this variation of the model:

$$
\frac{\operatorname{var}\left(X \mid \mathcal{F}_{I}\right)^{-1}-\operatorname{var}\left(X \mid \mathcal{F}_{U}\right)^{-1}}{N^{-1} \sum_{i=1}^{N} \operatorname{var}\left(X \mid \mathcal{F}_{i}\right)^{-1}}=2 \tau c .
$$

It is again straightforward to check that limiting information acquisition equilibria must satisfy (8) for some $\lambda_{\beta}$. Moreover, in the diversifiable risk case we can solve (45) to obtain

$$
\lambda_{\beta}=\tau \sigma_{z} \sigma_{\epsilon} \sqrt{\frac{1}{2 \tau c}-\sigma_{\epsilon}^{2}}
$$

A simple comparison with the expressions from Theorem 1 yields the statement in the proposition on the increase in information acquisition. The results on information acquisition in the systematic risk model follow similarly from (45), see Cao (1999). Finally, taking limits in (44) one obtains the limiting option prices (21) and (22).

\section{Proof of Proposition 4.}

Following Kyle (1989), let us conjecture that the optimal trading strategies of the agents are linear, namely let $\theta_{i} \equiv r Y_{i}-q P_{N}$; for $i=1, \ldots, m$, and $\theta_{i} \equiv-w P_{N}$, for $i=m+1, \ldots, N$. It is straightforward to verify that, using our notation, the characterization in Theorem 5.2 of Kyle (1989) reduces to the following system of equations for $(d, r, q, w):{ }^{37}$

$$
\begin{aligned}
(m q+n w) d & =1 \\
(1-\phi(r))(1-q d) & =1-\eta(r) \\
r & =\left(\frac{1}{\tau \sigma_{\epsilon}^{2}}\right) \frac{(1-\phi(r))(1-2 \eta(r) d)}{(1-\eta(r) d)} \\
\eta(r) d-\gamma(r) & =w d \eta(r)\left(\frac{d}{1-w d}+\tau \operatorname{var}\left(X \mid \mathcal{F}_{U}\right)\right)
\end{aligned}
$$

where

$$
\eta(r)=r \sigma_{\epsilon}^{2} \operatorname{var}\left(X \mid \mathcal{F}_{I}\right)^{-1}
$$

\footnotetext{
${ }^{37}$ The coefficient $b$ is given as before by $b / d=r$. Also note that for clarity we drop the subscript $N$ from the expressions.
} 


$$
\begin{aligned}
\phi(r) & =\frac{r^{2}(m-1) \sigma_{\epsilon}^{2}}{(m-1) r^{2} \sigma_{\epsilon}^{2}+V_{z}^{2}} ; \\
\gamma(r) & =\left(\frac{\operatorname{var}\left(X \mid \mathcal{F}_{U}\right)}{\operatorname{var}\left(X \mid \mathcal{F}_{I}\right)}\right) \frac{\sigma_{\epsilon}^{2} r^{2} m}{\left(m r^{2} \sigma_{\epsilon}^{2}+V_{z}^{2}\right)}
\end{aligned}
$$

with $\operatorname{var}\left(X \mid \mathcal{F}_{I}\right)$ and $\operatorname{var}\left(X \mid \mathcal{F}_{U}\right)$ given by the expressions (28) and (29).

As before, for notational convenience we have dropped the subscript $N$ from the pricing variables. Now let's assume that (4) holds, and conjecture (8). If $\alpha=\beta>0$, we immediately have from (47) that $\lim _{N \uparrow \infty} w_{N} d_{N}=0$. From (50) we then get that $\lim _{N \uparrow \infty} \eta\left(r_{N}\right)=0$, which in turns implies that $\lim _{N \uparrow \infty} q_{N} d_{N}=0$ from (48), since $\lim _{N \uparrow \infty} \phi\left(r_{N}\right)=0$. Finally, we have that $\lim _{N \uparrow \infty} r_{N}=1 /\left(\tau \sigma_{\epsilon}^{2}\right)$. Some straightforward calculations show that $\lim _{N \uparrow \infty} d_{N} N^{\beta}=d_{\beta}$, as given in Theorem 1. Furthermore, one can verify that the limiting trading strategies for both informed and uninformed coincide in the perfect competition and the imperfect competition models, and thereby the expected utilities are given by our previous limiting expressions, and the endogeneously determined $\lambda_{\beta}$ is again the same as the one provided in Theorem 1.

This verifies that the equilibrium in the imperfectly competitive market with large noise and (8) does indeed converge to its perfectly competitive counterpart. We are left to check whether there are other limiting equilibria. One can verify, as in the proof of Lemma 1, that prices would become perfectly revealing or completely uninformative if $\alpha \neq \beta$, from which we can conclude, by comparing the expected utilities of informed and uninformed agents, that $\alpha=\beta$ is also a necessary condition for existence of a limiting economy with endogenous information acquisition in the imperfectly competitive model.

\section{Proof of Proposition 5.}

Take an economy $\mathcal{E}_{N}(\beta)$ with an arbitrary number of agents $N$. Consider information acquisition decision of two agents, one who chooses to become informed with risk-aversion parameter $\tau_{I}$, and a second who stays uninformed with risk-aversion parameter $\tau_{U}$. We will argue that in the limit $\tau_{U} \geq \tau_{I}$ by contradiction. Consider an equilibrium in which agents with $\tau_{I}$ become informed, but agents of risk-aversion $\tau_{U}$ do not, and $\tau_{I}>\tau_{U}$. We next show that this set of strategies cannot be an equilibrium for $N$ large enough. Note that in any equilibrium it must be the case that

$$
\begin{aligned}
& -\sqrt{\operatorname{var}\left(X \mid \mathcal{F}_{I}\right)} e^{\tau_{I} c} \geq-\sqrt{\operatorname{var}\left(X \mid \mathcal{F}_{U}\right)} ; \\
& -\sqrt{\operatorname{var}\left(X \mid \mathcal{F}_{U}\right)} \geq-\sqrt{\operatorname{var}\left(X \mid \mathcal{F}_{I}\right)} e^{\tau_{U} c} .
\end{aligned}
$$

Equation (51) simply states that the informed agent is better off by buying the signal than by not purchasing it, and (52) requires that the uninformed agent will not desire to buy a signal. 
Taking limits, from Theorem 1 we have that the above inequalities reduce to $e^{\tau_{I} c} \leq e^{\tau_{U} c}$, i.e. $\tau_{I} \leq \tau_{U}$, which is a contradiction. The above argument shows that any set of strategy profiles in which an agent $\tau_{\text {min }}$ does not acquire information, but an agent with risk-aversion $\tau_{i}>\tau_{\text {min }}$ does, cannot be an equilibrium for $N$ large enough. The rest of the proof follows the same lines as Theorem 1.

\section{Proof of Proposition 6.}

The proof is very similar to that of Theorem 1, so we simply sketch the necessary steps. First, we write out the equilibrium conditions fixing $m$ and $N$. Then, using the asymptotic approximation to $m_{N}^{*}$ we explicitly compute the limiting equilibria for $\alpha=1$ and $\alpha \neq 1$. The same arguments as before yield that the only limiting equilibria with information acquisition is that with $\alpha=1$. For simplicity in the exposition we assume $\tau=1$ in what follows (the extension to general $\tau$ is immediate).

The market clearing condition is given by

$$
\sum_{i=1}^{m} \frac{\mathbb{E}\left[X \mid Y_{i}, Z_{i}, P_{N}\right]-P_{N}}{\operatorname{var}\left(X \mid Y_{i}, Z_{i}, P_{N}\right)}+\sum_{i=m+1}^{N} \frac{\mathbb{E}\left[X \mid Z_{i}, P_{N}\right]-P_{N}}{\operatorname{var}\left(X \mid Z_{i}, P_{N}\right)}=\sum_{i=1}^{N} Z_{i}
$$

Define the projection coefficients $\mathbb{E}\left[X \mid Y_{i}, Z_{i}, P_{N}\right]=\psi_{Y} Y_{i}+\psi_{Z} Z_{i}+\psi_{P} P_{N}$, and $\mathbb{E}\left[X \mid Z_{i}, P_{N}\right]=$ $\alpha_{Z} Z_{i}+\alpha_{P} P_{N}$. Letting $\Lambda \equiv n\left(1-\alpha_{P}\right) \operatorname{var}\left(X \mid Z_{i}, P_{N}\right)^{-1}+m\left(1-\psi_{P}\right) \operatorname{var}\left(X \mid Y_{i}, Z_{i}, P_{N}\right)$, the equilibrium conditions reduce to

$$
\begin{aligned}
\frac{b_{i}}{d_{i}}=\frac{\psi_{Y}}{1-\psi_{Z}} ; & i=1, \ldots, m ; \\
d_{i} \Lambda=\left(1-\psi_{Z}\right) ; & i=1, \ldots, m ; \\
d_{i} \Lambda=\left(1-\alpha_{Z}\right) ; & i=m+1, \ldots, N .
\end{aligned}
$$

It is straightforward to check, by explicitely taking limits in the above system of equations, that if $m_{N}$ satisfies (8) then the price coefficients converge to those in Theorem 1. In particular we have that

$$
\begin{aligned}
\lim _{N \rightarrow \infty} \frac{b}{d_{i}} & =\frac{1}{\sigma_{\epsilon}^{2}} \\
\lim _{N \rightarrow \infty} d_{i} N^{1 / 2} & =d_{0.5 .} .
\end{aligned}
$$

Moreover, by equating the expected utilities of the uninformed and informed we again arrive at the same expressions as in Theorem 1. 


\section{References}

Admati, A. R., 1985, "A noisy rational expectations equilibrium for multi-asset securities markets," Econometrica, 53(3), 629-657.

Admati, A. R., and P. Pfleiderer, 1986, "A monopolistic market for information," Journal of Economic Theory, pp. $400-438$.

— , 1987, "Viable allocations of information in financial markets," Journal of Economic Theory, 43, 76-115.

— 1990, "Direct and indirect sale of information," Econometrica, 58(4), 901-928.

Ausubel, L., 1990a, "Insider trading in a rational-expectations economy," American Economic Review, pp. 1022-1041.

— , 1990b, "Partially-revealing rational-expectations equilibrium in a competitive economy," Journal of Economic Theory, pp. 93-126.

Back, K., and J. F. Zender, 1993, "Auctions of divisible goods: on the rationale for the Treasury experiment," Review of Financial Studies, 6(4), 733-764.

Banker, R. D., and S. M. Datar, 1989, "Sensitivity, precision, and linear aggregation of signals for performance evaluation," Journal of Accounting Research, 27(1), 21-39.

Black, F., 1986, "Noise," Journal of Finance, 41, 529-543.

Brennan, M. J., and H. H. Cao, 1996, "Information, trade, and derivative securities," Review of Financial Studies, 9(1), 163-208.

Brunnermeier, M. K., 2001, Asset pricing under asymmetric information. Oxford University Press.

Bushman, R., and R. Indjejikian, 1993, "Accounting income, stock price and managerial compensation," Journal of Accounting 83 Economics, pp. 3-23.

— , 1995, "Voluntary disclosures and the trading behavior of corporate insiders," Journal of Accounting Research, pp. 293-316.

Campbell, J. Y., S. J. Grossman, and J. Wang, 1993, "Trading volume and serial correlation in stock returns," The Quarterly Journal of Economics, 108(4), 905-939.

Cao, H., 1999, "The effect of derivative assets on information acquisition and price behavior in a rational expectations equilibrium," Review of Financial Studies, 12(1), 131-163. 
Demange, G., and G. Laroque, 1995, "Private information and the design of securities," Journal of Economic Theory, 65, 233-257.

DeMarzo, P. M., and C. Skiadas, 1998, "Aggregation, determinacy, and informational efficiency for a class of economies with asymmetric information," Journal of Economic Theory, 80, $123-152$.

Diamond, D. W., 1985, "Optimal release of information by firms," Journal of Finance, 40(4), 1071-1094.

Diamond, D. W., and R. E. Verrecchia, 1981, "Information Aggregation in a Noisy Rational Expectations Economy," Journal of Financial Economics, 9(0), 221-235.

— , 1982, "Optimal managerial contracts and equilibrium security prices," Journal of Finance, 37, 275-287.

Duffie, D., and R. Rahi, 1995, "Financial market innovation and security design: an introduction," Journal of Economic Theory, 65, 1-42.

Grossman, S., 1976, "On the efficiency of competitive stock markets where trades have diverse information," Journal of Finance, 31(2), 573-585.

Grossman, S. J., and J. E. Stiglitz, 1980, "On the impossibility of informationally efficient markets," American Economic Review, 70(3), 393-408.

Hellwig, M. F., 1980, "On the aggregation of information in competitive markets," Journal of Economic Theory, 22, 477-498.

Hong, H., and M. Shum, 2004, "Rates of information aggregation in common value auctions," Journal of Economic Theory, 116, 1-40.

Jackson, M., and I. Kremer, 2004, "The relationship between the allocation of goods and a seller's revenue," Journal of Mathematical Economics, 40, 371 - 392.

Jackson, M. O., 2003, "Efficiency and information aggregation in auctions with costly information," Review of Economic Design, 8(2), 121-141.

Kim, O., and R. E. Verrecchia, 1991, "Trading volume and price reaction to public announcements," Journal of Accounting Research, 29(2), 302-321.

Kremer, I., 2002, "Information aggregation in common value auctions," Econometrica, 70(4), $1675-1682$.

Kyle, A. S., 1985, "Continuous auctions and insider trading," Econometrica, 53(6), 1315-1336. 
— 1989, "Informed speculation with imperfect competition," Review of Economic Studies, 56(3), 317-355.

Matthews, S., 1984, "Information acquisition in discriminatory auctions," in Bayesian models in economic theory, ed. by M. Boyer, and R. Kihlstrom. chap. 11, pp. 181-207.

Milgrom, P. R., 1979, "A convergence theorem for competitive bidding with differential information," Econometrica, 47(3), 679-688.

— , 1981, "Rational expectations, information acquisition and competitive bidding," The Quarterly Journal of Economics, 140(2), 341-388.

Morrison, A. D., and N. Vulkan, 2003, "Making money out of publicly available information," working paper, University of Oxford.

Pesendorfer, W., and J. M. Swinkels, 1997, "The loser's curse and information aggregation in common value auctions," Econometrica, 65(6), 1247-1281.

— , 2000, "Efficiency and information aggregation in auctions," American Economic Review, 90(3), 499-525.

Rahi, R., 1996, "Adverse selection and security design," Review of Economic Studies, 63, $287-300$.

Swinkels, J., 1999, "Asymptotic efficiency for discriminatory private value auctions," Review of Economic Studies, 66, 509 - 528.

— , 2001, "Efficiency of large private value auctions," Econometrica, 69, 37 - 68.

Verrecchia, R., 2001, "Essays on disclosure," Journal of Accounting 6 Economics, pp. 97-180.

Verrecchia, R. E., 1982, "Information acquisition in a noisy rational expectations economy," Econometrica, 50(6), 1415-1430.

Viswanathan, S., J. J. Wang, and T. P. Witelski, 2001, "Optimal bidding in multi-unit discriminatory auctions: two bidders," working paper, Duke University.

Vives, X., 1988, "Aggregation of information in large cournot markets," Econometrica, 56(4), $851-876$.

Wang, J., 1993, "A model of intertemporal asset prices under asymmetric information," Review of Economic Studies, 60(2), 249-282.

Wilson, R., 1968, "On the theory of syndicates," Econometrica, 36, 119-132. 
Wilson, R., 1977, "A bidding model of perfect competition," Review of Economic Studies, $44(3), 511-518$.

— , 1979, "Auctions of shares," The Quarterly Journal of Economics, 93(4), 675-689. 

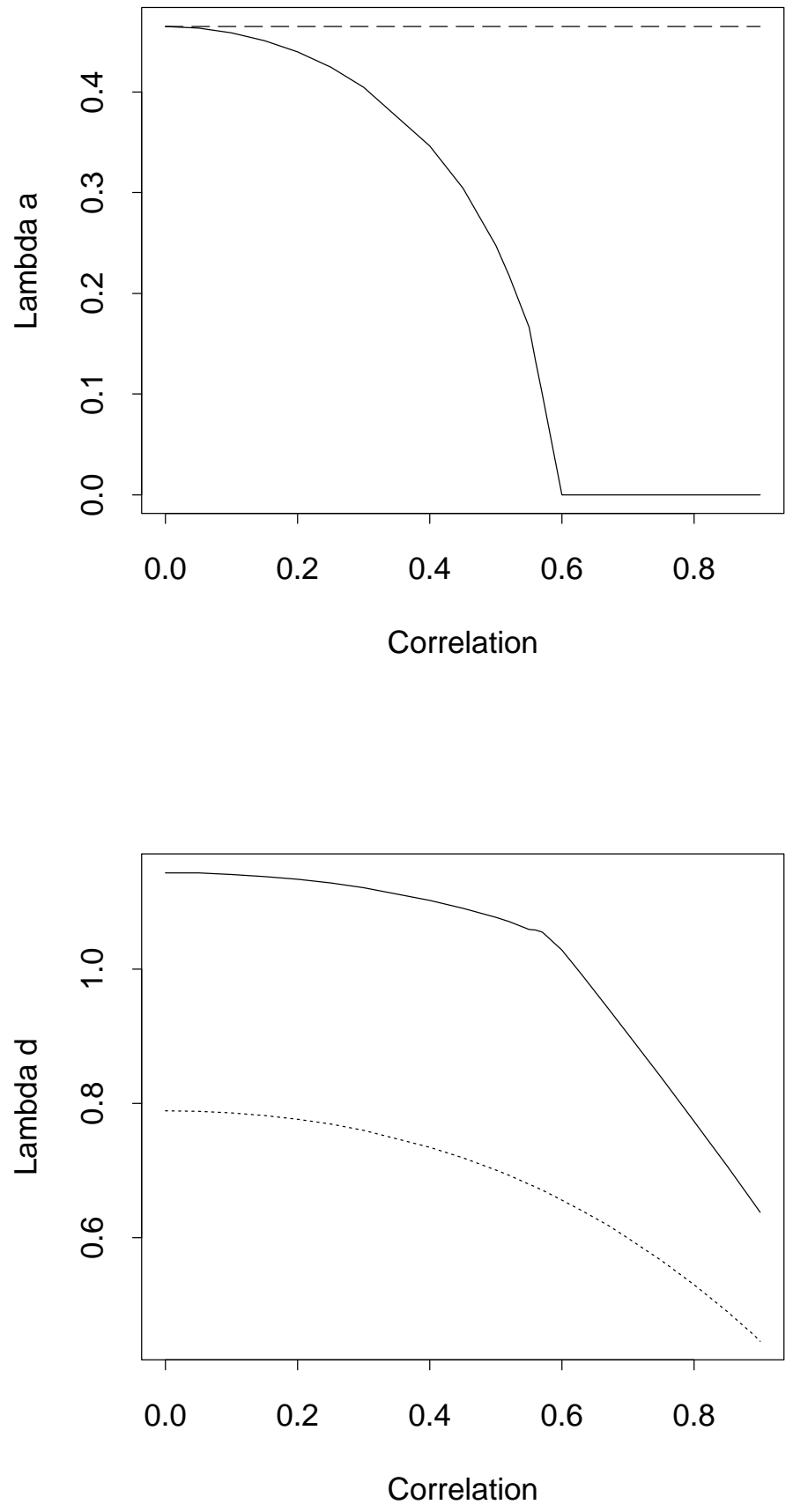

Figure 1: Equilibrium values $\lambda_{a}(\beta)$ (top graph) and $\lambda_{d}(\beta)$ (bottom graph) for the mixed model (solid lines), systematic risk model (dotted line), and in the one-asset economy (dashed line). Parameter values: $c_{a}=c_{b}=0.3, \delta=0.2, \tau=\sigma_{\epsilon}^{2}=\sigma_{z}^{2}=1$. 